\title{
Reducing the risk of pet-associated zoonotic infections
}

\author{
Jason W. Stull VMD PhD, Jason Brophy MD MSc, J.S. Weese DVM DVSc
}

See also page 715 and www.cmaj.ca/lookup/doi/10.1503/cmaj.150274

CMAJ Podcasts: author interview at soundcloud.com/cmajpodcasts/zoonotic-infections

Competing interests: None declared.

This article has been peer reviewed.

Correspondence to: Jason W. Stull, stull.82@osu.edu

CMAJ 2015. DOI:10.1503 /cmaj.141020
$\mathrm{P}$ et ownership can have health, emotional and social benefits; however, pets can serve as a source of zoonotic pathogens. One large, regional survey reported more than $75 \%$ of households having contact with a pet, ${ }^{1}$ and close, intimate interactions with pets (e.g., sleeping in beds with owners, face licking) are common., ${ }^{1,2}$ Additional surveys suggest that the general public and people at high risk for pet-associated disease are not aware of the risks associated with highrisk pet practices or recommendations to reduce them; for example, $77 \%$ of households that obtained a new pet following a cancer diagnosis acquired a high-risk pet. ${ }^{1,3}$ This statistic is not surprising - studies suggest physicians do not regularly ask about pet contact, nor do they discuss the risks of zoonotic diseases with patients, regardless of the patient's immune status. ${ }^{1,3,4}$

We review human infections acquired from pets, their risk factors and means of prevention. We limit the discussion to pet species typically owned by the general public (i.e., dogs, cats, fish, birds, amphibians, reptiles, rabbits and other rodents). Few systematic reviews or robust epidemiologic studies exist on this topic; most of our evidence comes from consensus guidelines and best practices for specific high-risk groups, with extrapolation to others (Box 1).

\section{How are pet-associated infections transmitted?}

People may acquire pet-associated zoonotic infections through bites, scratches or other direct

\section{- Ker points}

- Pets are a potential source of infection, with patients who are immunocompromised, pregnant women, young children and older adults at the greatest risk for transmission.

- Proper pet selection (species, age), pet care and personal (hand) hygiene practices can reduce disease risk.

- Physicians and other health care providers may, with the guidance of existing resources, counsel patients on safe pet ownership and contact to reduce pet-associated disease. contact of the skin or mucous membranes with animals, contact with animal saliva, urine and other body fluids or secretions, ingestion of animal fecal material, inhalation of infectious aerosols or droplets and through the bite of arthropods and other invertebrate vectors. ${ }^{5}$ Through these mechanisms, companion animals are a potential source for more than 70 human diseases, ${ }^{2,5,6}$ but this number is likely an underestimate given the molecular and epidemiologic evidence of the interspecies exchange of pathogens, such as multidrug resistant bacteria. ${ }^{7}$

Patient surveys and epidemiologic studies on the topic suggest that the occurrence of petassociated disease is low overall. ${ }^{1,8}$ Owing to a relative absence of reportable pathogens and complicating factors (e.g., non-pet exposure pathways, frequent subclinical shedding by pets), the proportion of human disease attributable to pets is unknown, and any reported frequency of such infections is likely underestimated. Yet, pet contact has been identified as a risk factor for many diseases, with case-control studies and molecular typing data strongly supporting pet sources for bacterial (e.g., Campylobacter, Salmonella), fungal (e.g., dermatophytes), parasitic (e.g., Toxoplasma gondii) and viral pathogens (e.g., lymphocytic choriomeningitis virus) ${ }^{6,9-12}$ Although pets do not typically directly transmit arthropod-borne diseases to people (e.g., Lyme borreliosis, ehrlichiosis, anaplasmosis), they do bring the zoonotic disease vectors - ticks and fleas - in close proximity to people, potentially increasing disease risk.

\section{Who is most likely to acquire a pet-associated infection?}

Despite the small role pets are likely to play in the overall transmission of pathogens, disease risk is not uniform; pet (e.g., species, age), management (e.g., housing) and patient factors influence risk. Based on cohort and case-control studies, young children (age $<5 \mathrm{yr}$ ) and older adults (age $\geq 65 \mathrm{yr}$ ), patients who are immunocompromised and women 
who are pregnant are at increased risk for zoonotic diseases, may have more severe disease, may have symptoms for a longer duration, or may have more severe complications than other patients. ${ }^{13-15}$

The immune-related mechanisms for increased disease risk are incomplete immune development, waning immune response, temporary hormone-induced immune suppression, such as in pregnancy, or congenital or acquired immunodeficiencies (e.g., metabolic diseases and cancer). ${ }^{5}$ In addition, children (notably those aged 3-5 yr) and some people with developmental disabilities may have suboptimal hygiene practices or higher risk contacts with animals that further increase risk. ${ }^{2}$ Furthermore, the specific immune deficiency may increase risk for particular pathogens; for example, newborn infants may be at increased risk of invasive salmonellosis, and pregnant women may be more likely to acquire lymphocytic choriomeningitis. However, this area is poorly understood. ${ }^{16}$

\section{Which pet-associated pathogens are of the greatest concern?}

Although many pathogens can be transmitted from pets to people, the pathogens of particular concern are less numerous (Box 2). The pathogens of greatest concern are described below.

\section{Bacterial diseases}

\section{Bartonella species}

Bartonellosis often induces lymphadenopathy and fever in patients with competent immune systems. More severe disease (e.g., bacteremia, endocarditis, neuroretinitis and proliferative lesions on the skin, liver or spleen) can occur in high-risk patients. ${ }^{17}$ Cats (especially juveniles) are the reservoir for Bartonella clarridgeiae and Bartonella henselae, with transmission most commonly occurring from a cat scratch (claws can become contaminated with feces from infected fleas) or flea bite. ${ }^{17}$

\section{Campylobacter jejuni}

Self-limiting diarrhea, vomiting and fever are common in Campylobacter jejuni infection. In high-risk patients, septicemia and diarrhea (with relapses) may be seen. Several pet species can transmit $C$. jejuni, most notably dogs and cats, passing infectious organisms in their feces. Juvenile dogs and cats are more likely to shed Campylobacter species than their mature counterparts, and recent acquisition of a puppy or kitten is associated with the highest risk of transmission. ${ }^{9,18}$

\section{Capnocytophaga canimorsus and Pasteurella multocida}

These organisms are common commensals in the oral cavity of dogs and cats. Transmission generally occurs through the bite of an infected or colonized animal or contact with saliva (such as by licking) on mucous membranes or an open wound. In patients at high risk, severe wound infections, sepsis, disseminated intravascular coagulation or death can occur. Patients with no spleen, older adults and people with alcohol dependence are at particularly increased risk for infection with Capnocytophaga canimorsus. ${ }^{19}$

\section{Multidrug-resistant bacteria}

Multidrug-resistant bacteria of public health importance have been found in people and companion animals. ${ }^{20,21}$ Cross-sectional studies have shown pet owners to have a six-fold greater risk for colonization with extended-spectrum $\beta$-lactamase-producing Escherichia coli than people who do not own pets, ${ }^{22}$ and pets owned by people with compromised immune systems are more likely to be colonized with Clostridium difficile than those owned by people with competent immune systems. ${ }^{20} \mathrm{~A}$ similar increased risk was seen in dogs that visit human health care facilities, with acquisition of $C$. difficile and methicillin-resistant Staphylococcus aureus two to five times more common than in dogs involved in other interventions. ${ }^{21}$ The current thinking for these predominantly human pathogens is that people serve as the main reservoir, but that household pets become colonized or infected, thus serving as a secondary source for human infection. ${ }^{7}$

\section{Salmonella species}

In immunocompetent people, salmonellosis most often results in self-limiting gastrointestinal disease, although serious disease can develop. The disease can be more severe in patients at high risk, resulting in bacteremia or serious systemic and localized infections, such as meningitis (in newborns) and osteomyelitis (in patients with sickle cell anemia). Although many pet species have been implicated in human disease, amphibians,

\section{Box 1: Evidence used in this review}

We identified primary literature, reviews and consensus guidelines through PubMed using the following search terms: "zoonosis," "human AND infection," "pet," "companion AND animal." We then performed searches of cited references on key articles. We identified more than 500 articles of interest, of which we included 332 articles in this review based on their content. Most of the identified articles were case reports or series or crosssectional studies, with limited studies involving cohort or case-control designs. We identified several consensus guidelines and reviews, primarily focused on high-risk groups. 
reptiles, exotic animals, rodents and young poultry pose the greatest risk. Reptiles and amphibians are estimated to be responsible for $11 \%$ of all sporadic Salmonella infections among patients less than 21 years of age, ${ }^{11}$ and direct contact with such animals is not required for zoonotic transmission. In one study, $31 \%$ of reptile-associated salmonellosis cases occurred in children less

\section{Box 2: Pathogens of particular concern in pet-associated infections}

Disease in high-risk patients (age $<5$ or $\geq 65 \mathrm{yr}$, immunocompromised or pregnant)

Pathogen

Key pet sources

Incidence

Severity

\section{Bacterial diseases}

Bartonella species

Brucella canis

Campylobacter jejuni

Capnocytophaga canimorsus

Chlamydophila psittaci

Leptospira interrogans

Multidrug-resistant bacteria

(e.g., MRSA, Clostridium difficile,

ESBL-producing organisms)

Mycobacterium marinum

Pasteurella multocida

Salmonella species

\section{Parasitic diseases}

Cutaneous larva migrans

(hookworms; canine and feline)

Cryptosporidium species

Echinococcus species

Giardia duodenalis

Dog, cats
Dogs, cats

Cats (B. clarridgeiae, $B$. henselae); rodents, rabbits, and dogs ( $B$. alsatica, $B$. vinsonii species)

\begin{tabular}{lll} 
Dogs & Rare & Moderate \\
$\begin{array}{l}\text { Dogs, cats (likely other } \\
\text { species) }\end{array}$ & High & Low \\
\hline
\end{tabular}

Dogs, cats Rare High

Birds Rare

Dogs, cats, rodents Low

Likely all species (although Variable

Moderate

data limited)

Fish
Dogs, cats
All species; high prevalence
in amphibians, reptiles,
exotic animals, rodents and
young poultry, in addition to
certain raw pet foods (e.g.,
meat, eggs and animal
product treats, such as pig's
ears)

Dogs, cats (particularly

Rare

Moderate

Moderate

Moderate

Variable
Low

\section{Moderate}

Moderate (particularly in newborns and patients with sickle cell anemia)

\begin{tabular}{|c|c|c|}
\hline $\begin{array}{l}\text { Ocular or visceral larva migrans } \\
\text { (roundworms; Toxocara canis } \\
\text { [dogs] and T. cati [cats]) }\end{array}$ & $\begin{array}{l}\text { Dogs, cats (particularly } \\
\text { juvenile animals) }\end{array}$ & $\begin{array}{l}\text { Low to mc } \\
\text { on geogra }\end{array}$ \\
\hline Toxoplasma gondii & $\begin{array}{l}\text { Cats (although food and } \\
\text { environment are main } \\
\text { sources) }\end{array}$ & Moderate \\
\hline
\end{tabular}

\section{Fungal diseases}

Dermatophytes (Microsporum canis, Trichophyton mentagrophytes)

Viral diseases
Cats (other species possible, High but less common)

Rodents (particularly mice Rare
juvenile animals) Dogs, cats, possibly birds

Dogs, cats

Low to high (depending on Low geography)

Moderate Moderate

Rare High

Moderate (species-specific Low assemblages; some shared by people and animals)

Low to moderate (depending Low to high (particularly among children)

High (particularly among pregnant women and very immunocompromised patients)

\section{Low}

Lymphocytic choriomeningitis and hamsters) Rare

and hamsters)

)

\author{
Any mammal (particularly Rare \\ unvaccinated cats and dogs)
}

Moderate to high (particularly among pregnant women and immunocompromised patients)

$\begin{array}{llll}\text { Rabies } & \begin{array}{l}\text { Any mammal (particularly } \\ \text { unvaccinated cats and dogs) }\end{array} & \text { Rare } & \text { High }\end{array}$

Note: ESBL = extended-spectrum $\beta$-lactamase, MRSA = methicillin-resistant Staphylococcus aureus. 
than 5 years of age and $17 \%$ occurred in children aged 1 year or younger; these findings highlight the heightened risk in children and the potential for reptile-associated Salmonella to be transmitted without direct contact with the animal or its enclosure. ${ }^{12}$ Outbreaks of pet-associated salmonellosis involving hedgehogs, rodents, young poultry, frogs and turtles have recently been reported, in which children accounted for a high proportion of cases $(35 \%-70 \%){ }^{23}$ In addition, various animal foods (e.g., raw meat, raw eggs and raw treats such as pig's ears) are commonly contaminated with Salmonella species. The feeding of these products are well-established risk factors for salmonellosis in pets, and associated human outbreaks have been identified..$^{24,25}$

\section{Parasitic diseases}

\section{Cryptosporidium species and Giardia duodenalis}

Subclinical or self-limiting diarrhea is generally observed with cryptosporidiosis and giardiasis, with weight loss and chronic diarrhea in highrisk patients. For cryptosporidiosis, symptoms may vary with the species or genotype of infection. Although most Giardia assemblages are species-specific, several are found in both animals and people with documented zoonotic transmission. Several pet species may harbor zoonotic Cryptosporidium and Giardia, including dogs and cats, which can pass the organisms in feces.

\section{Toxocara species}

Toxocara (roundworm) infection in humans typically involves subclinical or self-limited disease, but ocular or visceral larva migrans disease may develop in a small subset of patients. The highest risk is in young children owing to an increased likelihood of high inoculum after the ingestion of dog or cat feces containing ova. ${ }^{26}$ Because most household pets are regularly dewormed and larvae require two to three weeks after being passed in feces to become infective, the risk of exposure is highest after contact with soil contaminated with waste from untreated or stray animals, such as in sandboxes, gardens or playing fields. ${ }^{2,26}$

\section{Toxoplasma gondii}

Subclinical or self-limited febrile illness and lymphadenopathy are the most commonly reported symptoms after infection with Toxoplasma gondii in immunocompetent patients. Toxoplasmosis is of greatest concern in previously non-immune pregnant women and immunocompromised patients, regardless of exposure status; in such patients, congenital defects and encephalitis or meningitis can occur. ${ }^{5,8}$ Cats serve as the definitive host for $T$. gondii; however, food and the environment are the main sources of infection for humans.

\section{Fungal diseases}

\section{Dermatophytes}

Microsporum canis and Trichophyton mentagrophytes (e.g., ringworm) are the principal dermatophyte species of zoonotic importance. Severe disease is uncommon in immunocompetent patients, but disseminated infections can occur in immunocompromised patients.

\section{How can pet-associated infections be prevented?}

\section{Immunocompetent patients}

For patients who are immunocompetent, not pregnant and between the ages of 5 and 64 years, the risk of pet-associated disease is small. Without specific accepted recommendations or wellcontrolled studies involving members of this population, proper pet husbandry and general hand hygiene after higher-risk activities (e.g., feces removal, care of a pet with a known or suspected infectious zoonotic pathogen or contact with a high-risk species, such as a reptile) are likely adequate.

Injuries and infections associated with animal bites are the greatest risks for this population. About 4.5 million people in the United States are bitten by dogs each year, with the highest rate among people less than 14 years of age. ${ }^{27}$ Dog bites are responsible for $25 \%$ of animal-related treat-and-release visits to emergency departments and $17 \%$ of animal-related admissions to hospital. ${ }^{27}$ Any pet may bite or scratch if it is in a stressful situation, threatened or startled; proper pet selection, training and education on safe pet handling are important in reducing this risk.

\section{Immunocompromised patients or other patients at increased risk}

Patients with a compromised or incompletely developed immune system, such as young children $(<5 \mathrm{yr})$, older adults $(\geq 65 \mathrm{yr})$, pregnant women and patients with conditions or undergoing treatments that reduce immune function are at increased risk for pet-associated disease. ${ }^{5,15}$ However, pet ownership practices and the frequency of animal contact in this group are typically similar to those seen in the general public. ${ }^{2,3}$ Pet ownership, and the species involved, in households with immunocompro- 
mised children and children aged less than 5 years are similar to households with immunocompetent children. ${ }^{2,3}$
Recommendations for animal ownership and contact have been published for patients in highrisk groups, ${ }^{5,828-35}$ and additional guidelines are

Box 3: Suggestions for reducing transmission of zoonotic pathogens from pets to patients at high risk

\section{Personal hygiene}

- Wash hands after handling animals or their environment; supervise hand-washing for children less than 5 years of age

- Protect skin from direct contact with animal feces by wearing vinyl or household cleaning gloves or using a plastic bag when cleaning up after a pet

- Avoid contact with animal-derived pet treats

- Promptly wash bites and scratches inflicted by animals

- Do not allow pets to lick open wounds, cuts or medical devices (e.g., intravascular catheters); pets may also be discouraged from licking the faces of young children and immunocompromised patients

- Wear gloves to clean aquariums; do not dispose of aquarium water in sinks used for food preparation

- Ensure playground sandboxes are kept covered when not in use

Types and ages of pets

- Avoid contact with dogs and cats less than 6 months of age or stray animals (avoid acquiring a cat $<1 \mathrm{yr}$ old), particularly in households with very young children or immunocompromised patients

- Avoid contact with animals with diarrhea

- Avoid contact with young farm animals (e.g., petting zoos)

- Avoid contact with reptiles, amphibians, rodents and baby poultry (chicks and ducklings), as well as anything that has been in contact with these animals; such animals should be kept out of the households of high-risk patients

- Reptiles, amphibians, rodents and baby poultry should not be permitted to roam freely through a home or living area and should be kept out of kitchens and food-preparation areas

- Exercise caution when playing with cats to limit scratches; keep cats' nails short (declawing is not recommended)

- When acquiring a new pet, mature animals from established vendors pose a lower risk than other types of animals

- Avoid contact with exotic pets and non-human primates

- When visiting other households or locations with pets, take the same precautions

- If immunocompromise is transient or variable, consider waiting to acquire a new pet until after the patient is on stable immune suppression; people who work with animals (veterinarians, laboratory workers, pet store employees, farmers or slaughterhouse workers) should alter work practices during periods of maximal immunosuppression

- Consider limiting contact with animals in medical settings (e.g., therapy and visitation animals)

Pet health and husbandry

- Ensure pets remain healthy with regular veterinary visits and preventive care, including steps to control and prevent parasites

- Keep cats indoors; change litter boxes daily; wear vinyl or household cleaning gloves during cleaning and wash hands immediately after

- Keep litter boxes away from kitchens or other areas where food preparation and eating occur

- Keep dogs confined when possible; walk on a leash to prevent hunting, coprophagia and garbage eating

- Feed only canned or dry commercial food or well-cooked home-prepared food; egg or meat products and treats should be cooked, and dairy-based products should be pasteurized

- Prohibit pet access to non-potable water, such as surface water or toilet bowls

- Spay or neuter pets to reduce the likelihood of pathogen transmission through reproductive tract secretions

- Routinely clean and disinfect animal contact surfaces (e.g., cages, feeding areas) and immediately after contact with high-risk species or raw animal-based food items; freshly mixed diluted household bleach (1 part bleach to 32 parts water) or similar household disinfectants (e.g., quaternary ammonium compounds) are adequate

- Clean bird cage linings daily and small rodent cages frequently; wear disposable gloves, with or without a surgical mask, during litter handling

- Regularly (e.g., weekly) launder pet bedding

- Seek veterinary care at the first sign of illness in an animal 
available for animal-assisted interventions in health care facilities. ${ }^{36}$ Given the health benefits of animal ownership and the reluctance of patients to give up their pets, resources highlight the importance of following specific precautions. Patients at high risk and their households should have increased vigilance of their pets' health and take precautions to reduce pathogen transmission. Because few animal vaccines effectively reduce the risks of zoonotic disease transmission, other methods are important to reduce pet-associated disease. Pet contact guidelines address personal hygiene, types and ages of animals, and pet health and husbandry practices (Box 3 ).

The roles some pet species play in human disease have been clearly identified, such as in the transmission of Salmonella. Reptiles, amphibians, rodents, exotic species, baby poultry and raw animal-based pet-food items should be excluded from the households of patients at high risk. Strict hand hygiene after contact with these species and food items is critical, as is the cleaning and disinfection of contact surfaces (e.g., areas used for housing or otherwise having contact with high-risk species; counter tops and other items after contact with high-risk food items). In lower risk households, an understanding of the risk of salmonellosis (and similar pet-associated zoonoses) and preventive measures (e.g., hand hygiene after contact with these animals or their environments) is needed.

\section{What role can health care practitioners play?}

A key component to successful disease prevention programs is ensuring that patients at risk are aware of their risk and receive accurate, timely advice on risk reduction (Box 4).

Although veterinarians should assist in aspects of risk reduction (e.g., offering information on pet husbandry and preventive health measures), health care providers may be in a position to provide information about petassociated diseases and safe pet ownership, particularly to people who do not own a pet but come into regular contact with one. Furthermore, veterinarians may not be aware of the immune status of a pet owner who should receive counselling on safe pet ownership. ${ }^{37}$

Physicians should obtain a history of contact with pets or other animals during consultation, particularly with patients at high risk. ${ }^{30}$ Given that households that did not formerly own a pet may acquire one, ${ }^{3}$ and newly acquired pets can pose an increased risk for pathogens and adverse effects (e.g., bites, scratches), routine requestion- ing may be appropriate. Physicians should inquire about the types of animals with which the patient has contact, the health of pets living with the patient and the use of zoonotic disease prevention measures.

The information gained from an animal contact history will allow physicians to compile a more complete list of differential diagnoses for patients with illness. Pets and people often share the same environment and similar vector exposures; the presence of concurrent sick pets may prove useful in diagnosing certain illness, as was previously highlighted by a case of Rocky Mountain spotted fever in a patient and her two dogs. ${ }^{38}$

\section{Box 4: Applying the results of this review in clinical practice} (fictional case)

A five-year-old girl with leukemia, which had been diagnosed five months earlier, was admitted to hospital with abdominal pain, diarrhea (one episode with blood) and a fever that had lasted 12 hours. She had received chemotherapy eight days earlier and had no history of recent contacts with people who were ill or possible ingestion of contaminated food. On examination, she was febrile (oral temperature $39^{\circ} \mathrm{C}$ ) and tachycardic (heart rate 150 beats $/ \mathrm{min}$ ) with generalized abdominal pain and tenderness; her blood pressure and respiratory rate were normal. Laboratory investigations showed leukopenia (leukocyte count 0.5 [normal 4.7-13.5] $\times 10^{9} \mathrm{cells} / \mathrm{L}$ ), neutropenia (neutrophil count 0.2 [normal $1.5-8.5$ ] $\times 10^{9}$ cells/L), anemia (hemoglobin 98 [normal 105-135] g/L) and thrombocytopenia (platelets 75 [normal 150-450] $\times 10^{9}$ cells/L). The patient's liver enzymes were mildly elevated (alanine transaminase 88 [normal 10-40] U/L, aspartate transaminase 75 [normal 8-45] U/L).

The patient was admitted with a diagnosis of febrile neutropenia and was given piperacillin-tazobactam and tobramycin intravenously, in addition to metronidazole orally for suspected Clostridium difficile infection.

Blood and stool cultures both grew Salmonella enterica serovar Typhimurium. The oncologist requestioned the family and found that the child's sister had acquired a pet turtle two months earlier. The family recalled that they were advised not to acquire any new pets after their daughter received her diagnosis; however, they thought that restricting her contact with the turtle (i.e., keeping it only in the sister's room and not allowing her to touch the animal) was enough to protect her. The older sister had developed a mild diarrheal illness two weeks after acquiring the turtle, which the parents recalled after prompting. The family cleaned the turtle's tank in the bathtub, and both girls bathed together in that same bathtub on a regular basis.

This fictional case shows several important characteristics of petassociated zoonotic disease in at-risk populations. First, despite having received counseling regarding pet acquisition, the family either did not recall or adhere to the advice, highlighting the need for both verbal and written anticipatory guidance regarding pet safety at and after the time of diagnosis of immune-compromising conditions. Second, the family did not volunteer information about the newly acquired pet at the time of their child's admission, potentially leading to a delayed diagnosis. Frequent requestioning about pet exposures at the time of illness can help lead to earlier diagnosis and treatment, and routine questioning about pet contacts can afford opportunities to mitigate disease risk before illness occurs. Finally, despite not having direct contact with the pet, the risk of disease transmission for atrisk patients still exists with household contact. In this patient's case, transmission could have occurred through several possible routes: contamination of household members' hands after contact with the turtle and inadequate hand hygiene prior to food preparation or personal care of the child; contamination of a shared space (i.e., the bathtub); or close contact (i.e., bathing) with the sister, who was likely asymptomatically shedding Salmonella in her stool. 


\section{Gaps in knowledge}

Surveys suggest that most veterinarians and physicians do not regularly discuss zoonotic disease risks with clients, patients or each other. ${ }^{4,37} \mathrm{~A}$ recent push from both fields (the "One Health" initiative[www.onehealthinitiative.com]) aims to reduce this professional gap. Physicians may find it particularly helpful to reach out to veterinarians for information on a specific pet's preventive care, zoonotic disease history, risk of transmission and disease risks for unusual species. In addition, developing a rapport with local veterinarians or those who specialize in infectious disease may be useful (e.g., interdisciplinary meetings, sharing contact information with veterinarians through patients).

Existing pet-contact recommendations are based on relatively limited data, human disease outbreaks and general concepts in infectious disease prevention. Whether such recommendations are appropriate for the level of risk is unknown, and information in assisting a patient to make an informed decision regarding the risks and benefits of pet contact is limited.

Studies quantifying the disease risks attributable to pets are needed. Observational study designs combined with molecular testing and typing methods will be helpful in identifying the proportion of human infectious disease for which pet contact is responsible, how this proportion differs for specific pathogens, whether there are identifiable risk factors important for transmission, how effective various practices are at reducing pet-associated disease, and what fraction of pet-associated disease is preventable.

\section{References}

1. Stull JW, Peregrine AS, Sargeant JM, et al. Household knowledge, attitudes and practices related to pet contact and associated zoonoses in Ontario, Canada. BMC Public Health 2012;12:553.

2. Stull JW, Peregrine AS, Sargeant JM, et al. Pet husbandry and infection control practices related to zoonotic disease risks in Ontario, Canada. BMC Public Health 2013;13:520.

3. Stull JW, Brophy J, Sargeant JM, et al. Knowledge, attitudes, and practices related to pet contact by immunocompromised children with cancer and immunocompetent children with diabetes. J Pediatr 2014;165:348-55.e2

4. Hill WA, Petty GC, Erwin PC, et al. A survey of Tennessee veterinarian and physician attitudes, knowledge, and practices regarding zoonoses prevention among animal owners with HIV infection or AIDS. J Am Vet Med Assoc 2012;240:1432-40

5. Mani I, Maguire JH. Small animal zoonoses and immuncompromised pet owners. Top Companion Anim Med 2009;24:164-74.

6. Chomel B. Emerging and re-emerging zoonoses of dogs and cats. Animals 2014;4:434-45.

7. Morris DO, Lautenbach E, Zaoutis T, et al. Potential for pet animals to harbour methicillin-resistant Staphylococcus aureus when residing with human MRSA patients. Zoonoses Public Health 2012;59:286-93.

8. Glaser CA, Angulo FJ, Rooney JA. Animal-associated opportunistic infections among persons infected with the human immunodeficiency virus. Clin Infect Dis 1994;18:14-24.

9. Mughini Gras L, Smid JH, Wagenaar JA, et al. Increased risk for Campylobacter jejuni and $C$. coli infection of pet origin in dog owners and evidence for genetic association between strains causing infection in humans and their pets. Epidemiol Infect 2013;141:2526-35.

10. Younus M, Wilkins MJ, Davies HD, et al. The role of exposures to animals and other risk factors in sporadic, non-typhoidal Salmonella infections in Michigan children. Zoonoses Public Health 2010;57:e170-6.

11. Mermin J, Hutwagner L, Vugia D, et al. Reptiles, amphibians, and human Salmonella infection: a population-based, case-control study. Clin Infect Dis 2004;38(Suppl 3):S253-61.

12. Whitten T, Bender JB, Smith K, et al. Reptile-associated salmonellosis in Minnesota, 1996-2011. [Epub ahead of print]. Zoonoses Public Health 2014; June 9 [Epub ahead of print].

13. Hung CC, Hung MN, Hsueh PR, et al. Risk of recurrent nontyphoid Salmonella bacteremia in HIV-infected patients in the era of highly active antiretroviral therapy and an increasing trend of fluoroquinolone resistance. Clin Infect Dis 2007;45:e60-7.

14. Gradel KO, Norgaard M, Dethlefsen C, et al. Increased risk of zoonotic Salmonella and Campylobacter gastroenteritis in patients with haematological malignancies: a population-based study. Ann Hematol 2009;88:761-7.

15. Kourtis AP, Read JS, Jamieson DJ. Pregnancy and infection. $N$ Engl J Med 2014;371:1077.

16. Meyer Sauteur PM, Relly C, Hug M, et al. Risk factors for invasive reptile-associated salmonellosis in children. Vector Borne Zoonotic Dis 2013;13:419-21.

17. Breitschwerdt EB. Bartonellosis: one health perspectives for an emerging infectious disease. ILAR J 2014;55:46-58.

18. Tenkate TD, Stafford RJ. Risk factors for Campylobacter infection in infants and young children: a matched case-control study. Epidemiol Infect 2001;127:399-404.

19. de Boer MG, Lambregts PC, van Dam AP, et al. Meningitis caused by Capnocytophaga canimorsus: when to expect the unexpected. Clin Neurol Neurosurg 2007;109:393-8.

20. Weese JS, Finley R, Reid-Smith RR, et al. Evaluation of Clostridium difficile in dogs and the household environment. Epidemiol Infect 2010;138:1100-4.

21. Lefebvre SL, Reid-Smith RJ, Waltner-Toews D, et al. Incidence of acquisition of methicillin-resistant Staphylococcus aureus, Clostridium difficile, and other health-care-associated pathogens by dogs that participate in animal-assisted interventions. J Am Vet Med Assoc 2009;234:1404-17.

22. Meyer E, Gastmeier P, Kola A, et al. Pet animals and foreign travel are risk factors for colonisation with extended-spectrum betalactamase-producing Escherichia coli. Infection 2012;40:685-7.

23. Gastrointestinal (enteric) diseases: selected multistate outbreak investigations linked to animals and animal products. Atlanta: Centers for Disease Control and Prevention; [updated 2014]. Available: www.cdc.gov/zoonotic/gi/outbreaks.html. (accessed 2014 Aug. 5).

24. Leonard EK, Pearl DL, Finley RL, et al. Evaluation of petrelated management factors and the risk of Salmonella spp. carriage in pet dogs from volunteer households in Ontario (20052006). Zoonoses Public Health 2011;58:140-9.

25. Human salmonellosis associated with animal-derived pet treats United States and Canada, 2005. MMWR Morb Mortal Wkly Rep 2006;55:702-5.

26. Lee RM, Moore LB, Bottazzi ME, et al. Toxocariasis in North America: a systematic review. PLoS Negl Trop Dis 2014;8:e3116.

27. Adams A, Sutton JP, Elixhauser A. Emergency department visits and hospitalizations associated with animal injuries, 2009. HCUP statistical brief \#134. Rockville (MD): Agency for Healthcare Research and Quality; 2012. Available: www.hcup-us.ahrq.gov /reports/statbriefs/sb134.pdf (accessed 2014 Aug. 5).

28. Angulo FJ, Glaser CA, Juranek DD, et al. Caring for pets of immunocompromised persons. Can Vet J 1995;36:217-22.

29. Trevejo RT, Barr MC, Robinson RA. Important emerging bacterial zoonotic infections affecting the immunocompromised. Vet Res 2005;36:493-506.

30. Pickering LK, Marano N, Bocchini JA, et al.; Committee on Infectious Diseases. Exposure to nontraditional pets at home and to animals in public settings: risks to children. Pediatrics 2008; 122:876-86.

31. Hemsworth S, Pizer B. Pet ownership in immunocompromised children - a review of the literature and survey of existing guidelines. Eur J Oncol Nurs 2006;10:117-27.

32. Yokoe D, Casper C, Dubberke E, et al. Safe living after hematopoietic cell transplantation. Bone Marrow Transplant 2009; 44:509-19.

33. Panel on Opportunistic Infections in HIV-Infected adults and adolescents. Guidelines for the prevention and treatment of opportunistic infections in HIV-infected adults and adolescents: recommendations from the Centers for Disease Control and Prevention, the National Institutes of Health, and the HIV Medicine Association of the Infectious Diseases Society of America. 
Available: http://aidsinfo.nih.gov/contentfiles/lvguidelines /adult_oi.pdf (accessed 2014 Aug. 5)

34. National Association of State Public Health Veterinarians Animal Contact Compendium Comittee. Compendium of measures to prevent disease associated with animals in public settings, 2013. J Am Vet Med Assoc 2013;243:1270-88

35. Avery RK, Michaels MG. Strategies for safe living after solid organ transplantation. Am J Transplant 2013;13(Suppl 4):304-10.

36. Lefebvre SL, Golab GC, Christensen E, et al.; Writing Panel of Working Group. Guidelines for animal-assisted interventions in health care facilities. Am J Infect Control 2008;36:78-85.

37. Grant S, Olsen CW. Preventing zoonotic diseases in immunocompromised persons: the role of physicians and veterinarians. Emerg Infect Dis 1999;5:159-63.

38. Elchos BN, Goddard J. Implications of presumptive fatal Rocky Mountain spotted fever in two dogs and their owner. J Am Vet Med Assoc 2003;223:1450-2.
Affiliations: The Ohio State University (Stull), College of Veterinary Medicine, Department of Veterinary Preventive Medicine, Columbus, Ohio; Children's Hospital of Eastern Ontario (Brophy), Ottawa, Ont.; Centre for Public Health and Zoonoses (Weese); Department of Pathobiology (Weese), Ontario Veterinary College, University of Guelph, Guelph, Ont.

Contributors: All of the authors contributed substantially to the conception and design of the review, the acquisition of data, and the drafting and revising of the article. All of the authors approved the final version submitted for publication and agreed to act as guarantors of the work.

Acknowledgements: J.S. Weese was supported in part by a Canadian Institutes of Health Research Canada Research Chair in Zoonotic Diseases.

\section{Resources}

- Chomel B. Emerging and re-emerging zoonoses of dogs and cats. Animals 2014;4:434-45. Available: www.mdpi.com/2076-2615/4/3/434

- Dog bite prevention. Schaumburg: American Veterinary Medical Association. Available: www.avma .org/public/pages/Dog-Bite-Prevention.aspx

- Gastrointestinal (enteric) diseases from animals. Atlanta: Centers for Disease Control and Prevention. Available: www.cdc.gov/zoonotic/gi/index.html

- Health pets, healthy people. Atlanta: Centers for Disease Control and Prevention. Available: www.cdc.gov/healthypets/index.html

- Home and recreational safety: dog bites. Atlanta: Centers for Disease Control and Prevention. Available: www.cdc.gov/HomeandRecreationalSafety/Dog-Bites/index.html

- National Association of State Public Health Veterinarians Animal Contact Compendium Committee 2013. Compendium of measures to prevent disease associated with animals in public settings, 2013. J Am Vet Med Assoc 2013;243:1270-1288. Available: http://avmajournals.avma.org/doi/full/10.2460/javma.243.9.1270

- Pets and the immunocompromised person. Washington: Medline Plus. Available: www.nlm.nih.gov /medlineplus/ency/article/003967.htm

- Preventing infections during cancer treatment: caring for your pet. Atlanta: Centers for Disease Control and Prevention Foundation. Available: www.preventcancerinfections.org/health-tip-sheet/caring-your-pet

- Weese JS. Resources - pets (information sheets on pet ownership, husbandry and associated zoonotic diseases for the public, kids, and physicians; sample animal contact history form for health care providers); Worms and germs blog. Guelph: Scott Weese. Available: www.wormsandgermsblog.com

- Weese JS, Fulford MB. Companion animal zoonoses. lowa: Wiley-Blackwell; 2011.

- Lefebvre SL, Golab GC, Christensen E, et al.; Writing Panel of the Working Group. Guidelines for animal-assisted interventions in health care facilities. Am J Infect Control 2008;36:78-85. Available: www.sciencedirect.com/science/article/pii/S019665530700781X

THE CMA'S 2015

NEW IN PRACTICE GUIDE Everything residents need to know before entering practice

cma.ca/newinpractice

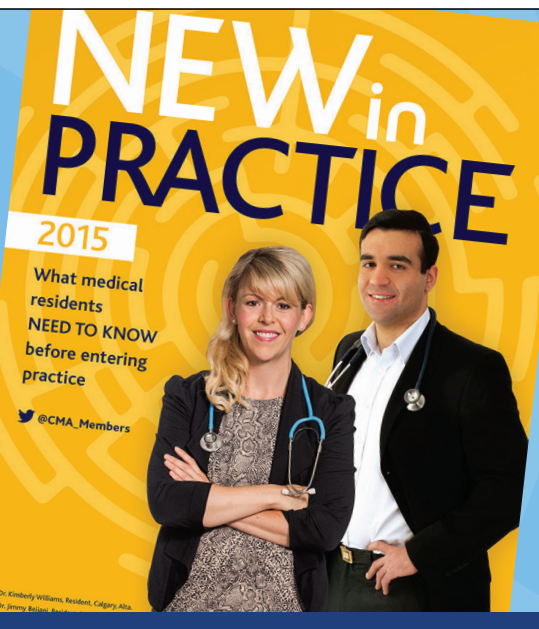

ASSOCIATION MÉDICALE CANADIENNE
CANADIAN MEDICAL ASSOCIATION 